\title{
Applications of Clinical Informatics to Child Mental Health Care: a Call to Action to Bridge Practice and Training
}

\author{
Juliet Edgcomb ${ }^{1} \cdot$ John Coverdale ${ }^{2} \cdot$ Rashi Aggarwal $^{3} \cdot$ Anthony P. S. Guerrero $^{4} \cdot$ Adam M. Brenner $^{5}$ \\ Published online: 17 February 2022 \\ (C) Academic Psychiatry 2022
}

A national state of emergency in children's mental health was declared by the American Academy of Pediatrics, American Academy of Child and Adolescent Psychiatry, and Children's Hospital Association on October 19, 2021 [1]. The COVID19 pandemic has disrupted the education of nearly all 55 million kindergarten through 12th grade students in the United States $[2,3]$. The recent US Surgeon General's Advisory on protecting youth mental health [4] underscores rising child mental health needs placing an unprecedented strain on the child mental health care system [5-7]. Together, these forces have been an impetus for child and adolescent psychiatrists and allied professionals to think critically about how to better leverage clinical informatics to meet the mental health needs of children [8] and learn collectively about the growing role of technology-driven and technology-informed modes of practice in supporting quality of child mental health care [9].

There have been two parallel trends over the last decade, both of which have been accentuated by the pandemic. The first is emotionally palpable to clinicians working with children and families: the rates of childhood mental health concerns and suicide have risen steadily throughout the decade [10]. By 2018, suicide reached the second leading cause of death among young people aged 10 to 34 years [11], and during 2020 there were dramatic increases in emergency

Juliet Edgcomb

JEdgcomb@mednet.ucla.edu

1 University of California Los Angeles Semel Institute for Neuroscience and Human Behavior, Los Angeles, CA, USA

2 Baylor College of Medicine, Houston, TX, USA

3 Rutgers New Jersey Medical School, Newark, NJ, USA

4 University of Hawai'i John A. Burns School of Medicine, Honolulu, HI, USA

5 University of Texas Southwestern Medical Center, Dallas, TX, USA department visits for suicide-related behavior among youth $[12,13]$. The second trend is an extremely rapid growth in health data, including patient access to data, and health care decision-making based on health information technology. However, the combination of rising child mental health care needs and the rapid advancement of digital health technologies is also coupled with a significant divide between child psychiatry and training in clinical informatics [14]. Notably, few child psychiatrists have had formal training or developed skillsets to adapt, develop, or critically evaluate the use of electronic health records (EHRs) at point of care, or to interpret the growing body of scientific literature leveraging EHRs to generate evidence for child psychiatry research [15].

This is a critical time for child mental health care. There is a growing recognition of the role of health data in changing clinical practice. There is also a crucial need to narrow the research to practice gap and to connect the traditionally siloed fields of child psychiatry and clinical informatics. In this context, we aim to discuss some of the potential applications of informatics to child mental health and to show how these two fields may be bridged in practice and training. We argue that better connecting these two fields could offer critical pathways for attending to the current crisis in child mental health. At the population level, improving the methods and infrastructure through which child mental health data are routinely collected and subsequently analyzed may help improve public health surveillance, identify inequities and gaps in access to care, and generate new knowledge in the discovery and management of child mental health conditions. At the individual child level, adapting the EHR as a tool for health delivery provides an important avenue to support measurement-based care, improve communication between providers, patients, and families, and mitigate clinician burnout and fatigue. However, the promise of bridging these fields must be tempered with an understanding of the feasibility within the context of workforce 
shortages, need for protection of health information, potential for biases, and challenges ahead.

\section{Clinical Informatics}

Clinical informatics is the application of informatics (i.e., the study of computational systems for data storage and retrieval) and information technology (i.e., computing technology used in the acquisition, storage, manipulation, display, and transmission of data) to deliver health care services [16, 17]. Clinical informatics was recognized in 2013 as a formal subspecialty by the American Board of Medical Specialties (ABMS) with over 1,800 board-certified physicians who have primary board certification in another specialty [18]. Physicians who practice clinical informatics collaborate with other health care and information technology professionals to design, implement, analyze, and evaluate information and communication systems to enhance individual and population health outcomes [17]. Physician informaticists leverage their knowledge of patient care combined with an understanding of informatics concepts and tools to lead measurement of patient and provider needs. They characterize, evaluate, and refine patient care processes, develop clinical decision support tools, and lead the design and continuous improvement of clinical information systems [19].

In its ideal form, clinical informatics functions to accelerate and support a learning health system, defined by the Institute of Medicine as a system "in which knowledge generation is so embedded into the core of the practice of medicine that it is a natural outgrowth and product of the healthcare delivery process and leads to continual improvement in care" [20,21]. This occurs through several iterative mechanisms including use of EHRs to systematically gather health data, generate evidence to improve care, and deploy point-of-care tools in turn informed by data and evidence [22, 23].

\section{EHR-Based Applications for Child Mental Health}

Since Torous et al. [24] advocated for training in clinical informatics in psychiatry, the field of mental health informatics has continued to grow, catalyzed by advancement of digital health technology and rising clinical needs. Examples of applications of clinical informatics in child psychiatry include point-of-care tools (e.g., embedding the Columbia-Suicide Severity Rating Scale in the clinician workflow of an EHR), patient-facing portals and patient access to medical records (e.g., open notes), public health surveillance and identification of areas of clinical need (e.g., tracking child mental health acute care utilization during the COVID-19 pandemic), and predictive analytics (e.g., leveraging large multi-site EHR datasets to inform personalized suicide risk prediction) (see Table 1).
At the onset of the COVID-19 pandemic, numerous studies leveraged data from EHRs to understand public health trends in child health. These in turn unearthed a disproportionate rise in child mental health need, inequalities in care delivery and access, and changes to health care delivery via telehealth $[12$, $13,26]$. Shifts in child mental health care delivery during the pandemic also brought a new need for provider tools such as EHR-telehealth integration, conversion of residual paper

Table 1 Examples of electronic health record-based applications for child mental health within a learning health system framework (adapted from the Heimdall Framework [25])

\section{Cohort discovery recognizes patients with similar attributes}

Example. The medical director of a clinic identifies the number of youths served by the clinic who have reported cannabis use (social history) or with a cannabis-use related diagnosis (diagnostic code)

2. Positive deviance distinguishes examples of better care against a benchmark

Example. After converting the firearms screening portion of an emergency psychiatric consultation note template from free text to a structured format, a health system measures screening completion rates

3. Negative deviance discovers examples of sub-optimal care

Example. A hospitalist receives a list of their patients receiving multiple concurrent antipsychotics and identifies opportunities to reduce polypharmacy

4. Predictive patient risk modeling leverages patterns in data to discover groups at higher risk of adverse outcomes

Example. A banner appears alerting a provider that a child has a high risk of a suicide attempt within 30 days and displays the predictors informing this risk calculation

5. Predictive care risk and outcome models identify situations that are at higher risk of poor care

Example. A health system monitors the rehospitalization rate of children who do and do not receive a referral for a follow-up appointment within 7 days of discharge after mental health hospitalization

6. Clinical decision support systems leverage algorithms applied to patient data to make specific practice recommendations

Example. A family nurse practitioner opens the health record of a toddler who recently screened positive on the Modified Checklist for Autism in Toddlers-Revised with Follow up (M-CHAT-R/F) and an alert appears prompting referral to a developmental pediatrician.

7. Comparative effectiveness research discovers the most effective treatments

Example. A researcher compares hospitalization rates among youth who had documentation of psychosocial care after initiation of an antipsychotic medication vs. youth prescribed antipsychotic medication without documentation psychosocial care

8. Intelligent assistance uses data to automate routine processes

Example. An electronic consent form auto-populates with patient identifying information, avoiding duplicative data entry

9. Case surveillance monitors data for outbreaks, areas of need, and treatment gaps

Example. A researcher combines aggregated deidentified health data across three health systems in a specific geographic area to measure local trends in youth suicide and self-harm during the COVID-19 pandemic 
documentation (e.g., consent forms) to EHR-integrated formats, increased communication between mental health and other medical providers, and flags and banners to identify isolation precautions and vaccination status [27].

While these efforts are laudable, the tremendous strain of the pandemic has also exposed the ways in which collection and analysis of health data is not yet adapted for child mental health care. For example, analysis of public health trends in child mental health using EHRs often relies on data stored in structured fields, most commonly, International Classification of Disease codes. However, structured data alone is often inaccurate in the discovery of mental health and suicide-related visits $[28,29]$. There has also been scarce adaptation of natural language processing and other text processing methods to detect child mental health conditions from large-scale EHRs [30]. Although diagnostic classification systems have supported the categorization of child mental health conditions from billing and diagnostic codes as exampled by the Child and Adolescent Mental Health Disorders Classification System [31], there is a lack of validated psychiatric comorbidity indexes for child mental health conditions equivalent to existing medical comorbidity indexes such as the pediatric complex chronic conditions (CCC) classification system [32].

In an article in this issue, Agrawal [33] quoted D.H. Lawrence, "What the eye doesn't see and the mind doesn't know, doesn't exist," in discussing the inclusion of social history components of the medical note. Kronsberg [34] also pointed to the rising need to educate trainees on social determinants of health. Information on the social history, for example, can become "invisible" at a population level when stored in unstructured (free text) fields of EHRs. Educational variables (grade, type of school, receipt of special education services) are almost entirely omitted from structured data fields, and developmental monitoring and screening measures are variably encoded in structured and unstructured fields. Commonly used structured EHR data elements and examples of elements potentially of interest to child psychiatrists are presented in Table 2.

In fact, common data models standardizing the storage and curation of data from EHRs have had scarce input from child mental health providers, and they often do not specify storage or encoding of social determinants of health relevant to child mental health care. In turn, it is difficult to leverage EHRs to answer questions about the role of school closures and child mental health utilization or about the disproportionate impact of social disruptions, adversity, and school-related mental health care gaps during the COVID-19 pandemic on children from communities of color. As social determinants of health are increasingly recognized as a core component of EHR data, meriting extraction and text analysis [36, 37], academic child psychiatrists have an important opportunity to use their clinical knowledge to improve systematic discovery of developmentally-specific antecedents of child mental health outcomes already being documented in EHRs).

To support a vision of a learning health system with the capacity to provide improved mental health care for children, there must be solid connections between collection of health data, development of clinically useful bedside tools to support quality of care, and communication between child health care providers. In the last decade, EHRs have evolved from tools predominantly used for documentation to interactive clinical decision-support systems that change care processes. A simple example is a clinician presented with a banner or flag that serves as a reminder a of national quality measure (e.g., a banner with the date a last weight was documented for a child treated with an antipsychotic), prompting adherence to the measure. With increasing use of measurement-based care, a larger number of standardized scales commonly used in psychiatry (e.g., the GAD-7, PHQ-9) and pediatrics (e.g., the MCHAT) are EHR-integrated [41, 42]. Notably, integration of these measures must be balanced with corresponding workflow infrastructure, such as by administration of measures through patient portals prior to visits or, perhaps, within a collaborative care framework, by another provider prior to referral. This growing link between health data collection, clinical workflows, and communication between providers supports the importance of collaborations in clinical informatics. Here is an opportunity for child psychiatrists to inform the development of clinically useful bedside tools and consider how these tools will generate data to support care in an integrated and scalable framework, particularly within primary care integrated contexts and collaborative care models emphasizing population health-oriented systems of care. Mobilization of health information across organizations, communities, and hospitals remains a barrier, and necessitates that child mental health providers have an awareness of efforts to improve health information exchange to further this vision.

In this issue, Aras [43] discusses the use of objective structured clinical examinations in measuring child psychiatry trainees' competency in communicating with children and families, highlighting the evolving challenge to assess live clinical performance. In practice, trainees are encouraged to learn the skill of "synchronous documentation," involving reviewing health records, writing clinical documentation, entering orders, resolving decision support prompts, and billing workflows, all while interfacing with the child and family inperson or through telehealth. As such, the model of "triadic communication" (clinician, patient, family) moves to the "quadratic" (clinician, patient, family, EHR). This is a chance for academic child psychiatrists not only to passively adopt these point-of-care tools traditionally developed from other specialties but also to learn the skills and procedures to proactively develop, refine, and adapt these tools to enhance communication and improve the quality of child mental health 
Table 2 Examples of types of structured versus unstructured (text) electronic health record data

\begin{tabular}{ll}
\hline Structured data & Unstructured data \\
Standardized & Patient characteristics \\
Demographics (sex, sexual orientation, gender identity, ethnicity, race, preferred language) & Medication adherence \\
$\begin{array}{l}\text { Encounter (admission and discharge, provider id, facility id, discharge disposition, admitting } \\
\text { source, payer type, facility type) }\end{array}$ & $\begin{array}{l}\text { Prior use of healthcare services } \\
\text { Substance use }\end{array}$ \\
$\begin{array}{l}\text { Diagnosis/condition (diagnosis id, encounter) } \\
\text { Procedure (procedure id, encounter) }\end{array}$ & Prior self-harm and suicide attempts \\
Vital signs & Exposure to bullying \\
Death (date and time, cause) & Exposure to adverse childhood experiences \\
Laboratory results & Family characteristics \\
Medications & Primary caregiver \\
Smoking status (tobacco) & Indicators of economic stability (income, caregiver \\
Non-standardized & employment) \\
Chief complaint & Child protective service involvement \\
Mental health screening scores (e.g., PHQ-9 & Environment \\
Psychiatric hold or involuntary hospitalization & Living arrangements \\
Family history & Food access \\
Geographic identifiers (e.g., FIPS & Transportation \\
Use of restraints or physical hold & Access to firearms \\
& Exposure to racism and discrimination \\
& Education and use of school-based services
\end{tabular}

\footnotetext{
${ }^{\text {a }}$ Examples adapted from [35]

${ }^{\mathrm{b}}$ Patient Health Questionnaire (PHQ-9)

${ }^{\mathrm{c}}$ Federal Information Processing Standards (FIPS), i.e., federal standard geographic codes
}

care. Physician engagement in the refinement of EHR-based tools has been suggested as one strategy to mitigate burnout, improve EHR proficiency, and increase satisfaction [44].

As clinical informatics accelerates, child psychiatry trainees must also be equipped to interpret the results of risk prediction models derived from health data and embedded in EHRs. Data collected from EHRs offer a promising opportunity for development and validation of risk prediction algorithms using a comprehensive set of potentially predictive variables. In theoretical alignment with the learning health system framework, implementation of risk models in the EHR enhances model usability by making results available to clinicians at point of care and impacting real-time decision-making. On the other hand, EHR-based data also have limitations with regard to data quality, completeness, and generalizability. Appropriate model calibration is contingent on clinical context. Perhaps the most promising application of EHR-based risk prediction is suicide risk assessment. An increasing number of health systems now have implemented suicidal behavior risk prediction models [45], which utilize machine learning and data analytics to process data from millions of individuals and, in turn, deliver a personalized risk prediction of suicidal behavior for an individual child [46]. Here again there is potential for child psychiatrists to participate in the development and implementation of these predictive tools. Input, advocacy, and iterative refinement from clinicians using these tools is imperative to their potential capacity to support quality child mental health care and clinical decision-making.

\section{Education and Training}

Education in child psychiatry training will need to provide trainees with the research literacy skills to understand the evidence base informing applications of clinical informatics in child psychiatry. These skills will become increasingly pertinent as trainees are exposed to clinical decision support and EHR-driven risk prediction in everyday practice. It is important that training support an awareness of potential areas of bias, for example, the potential for model miscalibration depending on clinical context, and racial and ethnic differences in the statistical performance of risk prediction models [47]. There is also a growing need for educators to teach trainees about aspects of privacy and confidentiality within the context of EHRs, including patient portals and transparent clinical documentation. Considerations for training and education in clinical informatics during and after child and adolescent psychiatry fellowship are presented in Table 3.

The October 2021 emergency declaration in children's mental health called for strategies to meet challenges in child mental health care through innovation and action [1]. How do psychiatric educators provide this generation of child psychiatrists with the skills to translate their clinical expertise to meaningful improvements in health information technology supporting child mental health care? Strategies to increase exposure to and training in clinical informatics must be tempered with recognition of clinical demands in already extended residency training programs. Most child psychiatry trainees are exposed to EHRs in daily practice and, increasingly, other 
Table 3 Considerations for training and education in clinical informatics during and after child and adolescent psychiatry fellowship

\section{General Curriculum Guidelines (all residents/fellows)}

Baseline-needs assessment at matriculation: computer skills, basic knowledge of Information Technology (IT) infrastructure, basic data science research literacy

Core didactics: Foundations in informatics, health data management, and informatics research literacy

Clinical training: Incorporate IT resources in clinical care, administrative, and teaching environments. Faculty preceptors discuss with trainees their use and adaptation of IT for clinical care and administration, along with areas of potential improvement and bias. Trainees are encouraged to bring forward areas of need and workflow improvement

\section{Pathways for Additional Training}

\section{Focused Experiences}

Elective rotation with hands-on project experience: Combines structured didactics with skill-building training modules, 2-4 weeks duration. Mentorship and guided readings from an informatics-trained clinician, access to EHR sandbox.

Example: Health Informatics Elective, Children's Hospital of Pennsylvania [38]

Resident-driven working groups:Trainee-led groups of interested residents and fellows organize extracurricular meetings to discuss resident workflow issues with potential EHR solutions. Faculty and clinical informatics fellows attend and provide mentorship and guidance.

Example: New EHR Resident Development Squad, Children's Hospital of Pennsylvania [38]

\section{Longitudinal Experiences}

Program-specific tracks: Monthly meetings with resident and physician informaticists, discussion of mental health specific applications of health IT and clinical Informatics. Mentorship from physician informaticists. May include independent program practicum.

Example: Clinical Informatics Track, University of North Carolina Psychiatry Residency Program [39]

Interdisciplinary adjunctive training: Concurrent elective training program with residents and fellows across medical and surgical fields. Involves longitudinal project, faculty mentorship, and access to EHR sandbox and programming support.

Example: UCLA Resident Informaticist Program [40]

Specialization [19]

Clinical Informatics Fellowship: Designed to develop leaders with expertise in biomedical informatics and health IT. Two-year program with experiential training in alignment with ACGME Guidelines. Fellows become board-eligible for certification in clinical informatics

Practice Pathway to Board Certification (through 2022): Pathway to board-certification for applicants who have not completed training through Clinical Informatics Fellowship. Requires time in practice which may be supplemented by graduate education in Biomedical informatics

\section{Current National Efforts to Promote Opportunities in Clinical} Informatics

American Medical Informatics Association Mental Health Working Group, American Academy of Child and Adolescent Psychiatry Health Information Technology Committee, American Psychiatric Association Committee on Mental Health Information Technology, American Academy of Pediatrics Council on Clinical Information Technology digital health technologies (e.g., mobile apps, patient portals) that inform and shape clinical training. Several psychiatry residencies have recently developed elective specialized "tracks" in clinical informatics, including the University of North Carolina and the University of California, Los Angeles [39]. These were developed, in part, to meet the rising interest of trainees. However, these mechanisms will likely only be accessed by a subset of trainees with a particular interest in this area.

Perhaps a starting point is to build a balanced awareness of the role of health information technology and informatics in the practice of child psychiatry. This discussion must include an awareness of the limitations and potential for biases and harm. In the spirit of learning health systems, trainees may first be encouraged to identify gaps and areas of need in their institution's EHR system (e.g., improving documentation of access to firearms). They may also be provided with a conceptual framework for how this health data is stored, presented to the clinician, and acted upon within a decision-support system (e.g., through development of a pediatric-specific clinical workflow for firearm safety counseling). They should also be educated on the potential of excessive EHR demands to promote burnout [48-51]. These exercises in critical thinking related to EHR tools at point-of-care and leveraging of health data for advancement of child mental health, represent a shift in the curriculum away from solely teaching EHR use as an onboarding activity or administrative skill and fosters a more comprehensive understanding of clinical informatics as an evolving component of a learning health care system to support child mental health.

Given the rapidity with which shifts toward health information technology have occurred, child psychiatry educators may wish to seek training opportunities for themselves through departments of clinical informatics, clinical and translational institutes, and computer science and computational psychiatry fora. These relationships may provide opportunities for bidirectional exchanges of knowledge. As clinical experts, child psychiatrists have much to contribute to ascertaining the clinical usefulness and real-world utility of many health technologies. In turn, child psychiatrists also have much to learn from their colleagues, particularly physician informaticists of other specialties, who have dedicated the last decade to the refinement of health information technology to meet the needs of their patient populations.

The COVID-19 pandemic has spurred the field of child and adolescent psychiatry to accelerate the adoption of health technologies to meet the needs of children and families. This shift in practice has also unearthed a gap between advances in technology and training in clinical child and adolescent psychiatry. There are many exciting opportunities to bridge the gap between the bench of health technology development and the bedside of child mental health care. In turn, seizing these opportunities has the potential to improve access to and the quality of child mental health care across the continuum of 
mental health promotion, prevention, and treatment. Looking forward, we must also seek opportunities to open pathways for interested trainees to pursue formal clinical informatics fellowship training and to develop a balanced awareness of the roles of clinical informatics when training child psychiatry fellows.

\section{Declarations}

Conflict of Interest The authors have no conflicts of interest.

\section{References}

1. American Academy of Pediatrics, American Academy of Child and Adolescent Psychiatry and Children's Hospital AssociationAAPAACAP-CHA Declaration of a national emergency in child and adolescent mental health. 2021. Available from: https://www.aap. org/en/advocacy/child-and-adolescent-healthy-mentaldevelopment/aap-aacap-cha-declaration-of-a-national-emergencyin-child-and-adolescent-mental-health/. Accessed 2021 Dec 6.

2. Verlenden JV, Pampati S, Rasberry CN, Liddon N, Hertz M, Kilmer G, Viox MH, Lee S, Cramer NK, Barrios LC, Ethier KA. Association of Children's mode of school instruction with child and parent experiences and well-being during the COVID-19 pandemic - COVID experiences survey, United States, October 8 November 13, 2020. MMWR Recomm Rep. 2021;70:369-76.

3. Viner RM, Russell SJ, Croker H, Packer J, Ward J, Stansfield C, Mytton O, Bonell C, Booy R. School closure and management practices during coronavirus outbreaks including COVID-19: a rapid systematic review. Lancet Child Adolesc Heal. 2020;4:397-404.

4. Murthy V. U.S. Surgeon General's advisory on protecting youth mental health. 2021.

5. Nash KA, Zima BT, Rothenberg C, Hoffmann J, Moreno C, Rosenthal MS, Venkatesh A. Prolonged emergency department length of stay for us pediatric mental health visits (2005-2015). Pediatrics. 2021;147.

6. Sarvet B, Gold J, Bostic JQ, Masek BJ, Prince JB, Jeffers-Terry M, Moore CF, Molbert B, Straus JH. Improving access to mental health care for children: the Massachusetts child psychiatry access project. Pediatrics. 2010;126:1191-200.

7. Burns BJ, Phillips SD, Wagner HR, Barth RP, Kolko DJ, Campbell Y, Landsverk J. Mental health need and access to mental health services by youths involved with child welfare: a national survey. J Am Acad Child Adolesc Psychiatry. 2004;43:960-70.

8. Golberstein E, Wen H, Miller BF. Coronavirus disease 2019 (COVID-19) and mental health for children and adolescents. JAMA Pediatr. 2020;174:819-20.

9. Heldt JP, Agrawal A, Loeb R, Richards MC, Castillo EG, DeBonis $\mathrm{K}$. We're not sure we like it but we still want more: trainee and faculty perceptions of remote learning during the COVID-19 pandemic. Acad Psychiatry. 2021;45:598-602.

10. Burstein B, Agostino H, Greenfield B. Suicidal attempts and ideation among children and adolescents in US emergency departments, 2007-2015. JAMA Pediatr. 2019; 173:598-600.

11. Hedegaard H, Curtin SC, Warner M. Increase in suicide mortality in the United States, 1999-2018. NCHS Data Brief 2020;1-8.

12. Krass P, Dalton E, Doupnik SK, Esposito J. US pediatric emergency department visits for mental health conditions during the COVID-19 pandemic. JAMA Netw Open. 2021;4:e218533.

13. Zima BT, Edgcomb JB, Rodean J, Bussing R. Early impact of the COVID-19 shift to remote learning on acute child mental health care in US Children's Hospitals. AACAP Sci Proc. J Am Acad Child Adolesc Psychiatry; 2021. p. S296.

14. Peters TE. Transformational impact of health information technology on the clinical practice of child and adolescent psychiatry. Child Adolesc Psychiatr Clin N Am. 2017;26:55-66.

15. Benson NM, Edgcomb JB, Landman AB, Zima BT. Leveraging clinical informatics to improve child mental health care. J Am Acad Child Adolesc Psychiatry. 2020;59:1314-7.

16. Detmer DE, Lumpkin JR, Williamson JJ. Defining the medical subspecialty of clinical informatics. J Am Med Inform Assoc. 2009;16:167-8.

17. Gardner RM, Overhage JM, Steen EB, Munger BS, Holmes JH, Williamson JJ, Detmer DE, for the AMIA Board of Directors. Core content for the subspecialty of clinical informatics. J Am Med Inform Assoc. 2009;16:153-7.

18. Lehmann CU, Gundlapalli AV, Williamson JJ, Fridsma DB, Hersh WR, Krousel-Wood M, Ondrula CJ, Munger B. Five years of clinical informatics board certification for physicians in the United States of America. Yearb Med Inform. 2018;27:237-42.

19. American Board of Preventative Medicine. Clinical Informatics. 2021. Available from: https://www.theabpm.org/become-certified/ subspecialties/clinical-informatics/. Accessed 2021 Nov 29.

20. Borsky AE, Flores EJ, Berliner E, Chang C, Umscheid CA, Chang SM. Next steps in improving healthcare value: AHRQ evidencebased practice center program-applying the knowledge to practice to data cycle to strengthen the value of patient care. J Hosp Med. 2019; 14:311.

21. Olsen L, Aisner D, McGinnis JM, Editors, Medicine R on E. The learning healthcare system: workshop summary. IOM Roundtable Evidence-Based Med. 2007.

22. Enticott J, Johnson A, Teede H. Learning health systems using data to drive healthcare improvement and impact: a systematic review. BMC Health Serv Res. 2021;21:1-16.

23. Kraft S, Caplan W, Trowbridge E, Davis S, Berkson S, Kamnetz S, Pandhi N. Building the learning health system: describing an organizational infrastructure to support continuous learning. Learn Heal Syst. 2017;1:e10034.

24. Torous J, Chan S, Luo J, Boland R, Hilty D. Clinical informatics in psychiatric training: preparing Today's trainees for the already present future. Acad Psychiatry. 2018;42:694-7.

25. McLachlan S, Potts HWW, Dube K, Buchanan D, Lean S, Gallagher T, Johnson O, Daley B, Marsh W, Fenton N. The Heimdall framework for supporting characterisation of learning health systems. J Innov Heal Inform. 2018;25:77-87.

26. DeLaroche AM, Rodean J, Aronson PL, Fleegler EW, Florin TA, Goyal M, et al. Pediatric emergency department visits at US children's hospitals during the COVID-19 pandemic. Pediatrics. 2021;147.

27. Carlson JL, Goldstein R. Using the electronic health record to conduct adolescent telehealth visits in the time of COVID-19. J Adolesc Health. 2020;67:157-8.

28. Bertoia ML, Zhou L, Cheng H, Liu N, Seeger JD, Spalding WM, et al. Identification of patients with suicidal ideation or attempt in electronic health record data. Pharmacoepidemiol Drug Saf. 2019;28:162.

29. Arias SA, Boudreaux ED, Chen E, Miller I, Camargo CA, Jones $\mathrm{RN}$, et al. Which chart elements accurately identify emergency department visits for suicidal ideation or behavior? Arch Suicide Res. 2019;23:382-90.

30. Carson NJ, Mullin B, Sanchez MJ, Lu F, Yang K, Menezes M, Cook BL. Identification of suicidal behavior among psychiatrically hospitalized adolescents using natural language processing and machine learning of electronic health records. PLoS One. 2019;14:1-14.

31. Zima BT, Gay JC, Rodean J, Doupnik SK, Rockhill C, Davidson A, Hall M. Classification system for international classification of 
diseases, ninth revision, clinical modification and tenth revision pediatric mental health disorders. JAMA Pediatr. 2020;174:620-2.

32. Feudtner C, Feinstein JA, Zhong W, Hall M, Dai D. Pediatric complex chronic conditions classification system version 2: updated for ICD-10 and complex medical technology dependence and transplantation. BMC Pediatr. 2014;14:1-7.

33. Agrawal H. Never see an adult again. Acad Psychiatry. 2021. https://doi.org/10.1007/s40596-021-01495-y.

34. Kronsberg H, Bettencourt AF, Vidal C, Platt RE. Education on the social determinants of mental health in child and adolescent psychiatry fellowships. Acad Psychiatry. 2020. https://doi.org/10.1007/ s40596-020-01269-y.

35. HL7 International. Common Data Models Harmonization [Internet]. 2019. Available from: https://build.fhir.org/ig/HL7/ cdmh/profiles.html. Accessed 28 Dec 2021.

36. Bejan CA, Angiolillo J, Conway D, Nash R, Shirey-Rice JK, Lipworth L, Cronin RM, Pulley J, Kripalani S, Barkin S, Johnson KB, Denny JC. Mining 100 million notes to find homelessness and adverse childhood experiences: 2 case studies of rare and severe social determinants of health in electronic health records. J Am Med Inform Assoc. 2018;25:61-71.

37. Tan M, Hatef E, Taghipour D, Vyas K, Kharrazi H, Gottlieb L, Weiner J. Including social and behavioral determinants in predictive models: trends, challenges, and opportunities. JMIR Med Inform. 2020;8:1-12.

38. Mai MV, Luo BT, Orenstein EW, Luberti AA. A model for clinical informatics education for residents: addressing an unmet need. Appl Clin Inform. 2018;9.

39. Vitiello E, Kane M, Hutto A, Hall A. Building for the future: the creation of a residency training track to foster innovation through clinical informatics in psychiatry. J Am Med Inform Assoc. 2020;27:1747-51.

40. Singer JS, Cheng EM, Baldwin K, Pfeffer MA, Bartlett JD, Bell DS, et al. The UCLA Health resident Informaticist program - a novel clinical informatics training program. J Am Med Inform Assoc. 2017;24:832-40.

41. Bruni T, LaLonde L, Maragakis A, Lee J, Caserta A, Kilbourne AM, Smith S, Orringer K, Quigley J, McCaffery H, Lancaster B. The use of electronic health record tools to improve evidence-based treatment of adolescent depression in primary care. Acad Pediatr. 2021;21:1195-202.
42. Campbell K, Carpenter KLH, Espinosa S, Hashemi J, Qiu Q, Tepper M, et al. Use of a digital modified checklist for autism in toddlers - revised with follow-up to improve quality of screening for autism. J Pediatr. 2017;183:133-139.e1.

43. Aras S, Serim DB. Performance-based assessment in child and adolescent psychiatry residency training. Acad Psychiatry. 2021. https://doi.org/10.1007/s40596-021-01481-4.

44. Sequeira L, Almilaji K, Strudwick G, Jankowicz D, Tajirian T. EHR "SWAT" teams: a physician engagement initiative to improve electronic health record (EHR) experiences and mitigate possible causes of EHR-related burnout. JAMIA Open. 2021;4.

45. Walsh CG, Johnson KB, Ripperger M, Sperry S, Harris J, Clark N, Fielstein E, Novak L, Robinson K, Stead WW. Prospective validation of an electronic health record-based, Real-Time Suicide Risk Model. JAMA Netw Open. 2021;4:e211428.

46. Su C, Aseltine R, Doshi R, Chen K, Rogers SC, Wang F. Machine learning for suicide risk prediction in children and adolescents with electronic health records. Transl Psychiatry. 2020;10:1-10.

47. Coley RY, Johnson E, Simon GE, Cruz M, Shortreed SM. Racial/ ethnic disparities in the performance of prediction models for death by suicide after mental health visits. JAMA Psychiatry. 2021;78: 726-34.

48. Domaney NM, Torous J, Greenberg WE. Exploring the association between electronic health record use and burnout among psychiatry residents and faculty: a pilot survey study. Acad Psychiatry. 2018;42:648-52.

49. Gardner RL, Cooper E, Haskell J, Harris DA, Poplau S, Kroth PJ, Linzer M. Physician stress and burnout: the impact of health information technology. J Am Med Inform Assoc. 2019;26:106-14.

50. Kroth PJ, Morioka-Douglas N, Veres S, Babbott S, Poplau S, Qeadan F, Parshall C, Corrigan K, Linzer M. Association of Electronic Health Record Design and use Factors with clinician stress and burnout. JAMA Netw Open. 2019;2:e199609.

51. Nguyen OT, Jenkins NJ, Khanna N, Shah S, Gartland AJ, Turner $\mathrm{K}$, et al. A systematic review of contributing factors of and solutions to electronic health record-related impacts on physician well-being. J Am Med Inform Assoc. 2021;28.

Publisher's Note Springer Nature remains neutral with regard to jurisdictional claims in published maps and institutional affiliations. 